\title{
Acquired Brown's syndrome in a patient with systemic lupus erythematosus
}

\author{
J L Alonso-Valdivielso, B Alvarez Lario, J Alegre López, M J Sedano Tous, \\ A Buitrago Gómez
}

\begin{abstract}
A 27 year old woman with systemic lupus erythematosus (SLE) developed vertical diplopia with an apparent bilateral inferior oblique muscle palsy, resulting in a limitation of elevation of the globe in adduction. It resolved with systemic steroid treatment. A transient tenosynovitis affecting the superior oblique tendons was the probable underlying pathological mechanism. This is the first described case of Brown's syndrome associated with SLE.
\end{abstract}

(Ann Rheum Dis 1993; 52: 63-64)

Brown's syndrome is characterised by limitation of elevation of the adducted eye. It is due to a mechanical limitation of movement of the superior oblique tendon. Clinically, it simulates a palsy of the inferior oblique muscle with vertical diplopia on upward and inward gaze. This disorder has occasionally been described in association with inflammatory collagen vascular diseases including rheumatoid arthritis, juvenile chronic arthritis, and Still's disease.

We report here the case of a patient with systemic lupus erythematous (SLE) who developed bilateral Brown's syndrome. To our knowledge this association has not previously been described.

\section{Case report}

The patient, a 27 year old woman, presented with a six day history of diplopia. She had a history of SLE which was first diagnosed four years before her present admission. She was then treated with prednisone by mouth and her disease went into remission and remained stable with a low daily dose of prednisone for three years, at the end of which the drug was stopped. One month before admission she had had fever and arthritis in her wrists and left ankle, which resolved on treatment with naproxen.

On admission, the patient was asymptomatic except for diplopia. This was at a maximum on upward and lateral gazes. Ophthalmological examination showed that she was unable to elevate her eyes completely when they were in the adducted position (figs 1 and 2). All other ocular movements were intact. Pupillary responses and funduscopic examination were normal. No clicking sound or sensation on movement of the eyes was elicited. The red glass test and a Hess chart were consistent with the ophthalmological examination. An edrophonium chloride (Tensilon) test was negative. Physical examination was otherwise normal.
Laboratory studies showed a haemoglobin concentration of $105 \mathrm{~g} / \mathrm{l}$, a leucocyte count of $3.6 \times 10^{9} / 1$ with $29 \%$ lymphocytes, a platelet count of $440 \times 10^{9} / 1$, and erythrocyte sedimentation rate of $56 \mathrm{~mm} /$ hour (Westergren). Plasma protein electrophoresis showed a slight increase in the $\alpha_{2}, \beta$, and $\gamma$ globulins.

Serum complement component C3 was 610 $\mathrm{mg} / \mathrm{l}$ (normal 550-1200) and C4 was $140 \mathrm{mg} / \mathrm{l}$ (normal 200-500). Serological findings included a positive antinuclear antibody titre (1/640) and a positive titre of antibodies to double stranded DNA (1/160). IgG anticardiolipin antibody was positive using a enzyme linked immunosorbent assay (ELISA). Coagulation studies were compatible with the presence of lupus anticoagulant. Results of the following studies were within normal limits: biochemical profile, urine analysis, thyroid function, Veneral Disease Research Laboratory (VDRL) test, rheumatoid factor, antibodies to Ro (SS-A), La (SS-B), Sm, RNP, and cerebrospinal fluid analysis. Computed tomography and magnetic resonance imaging of her orbits and head showed no abnormality.

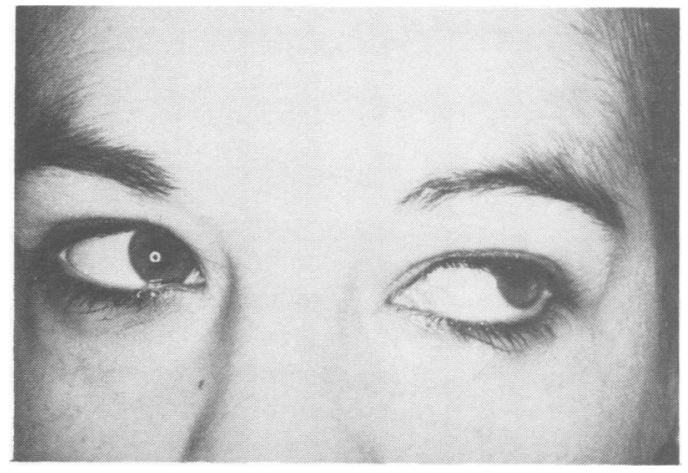

Figure 1 Limitation of elevation of the right eye in adduction.

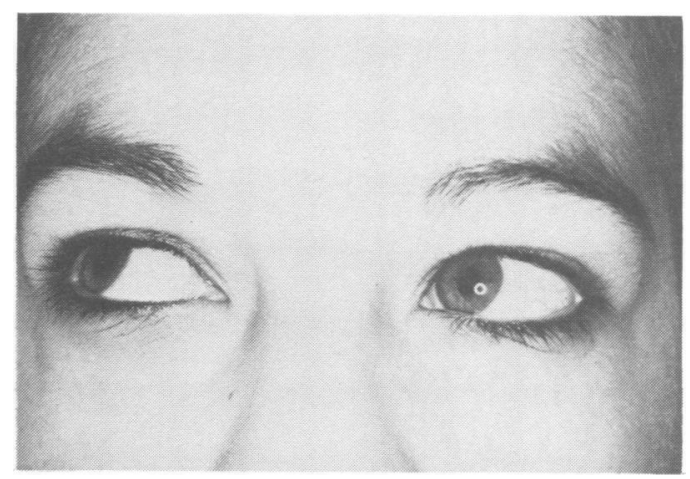

Figure 2 Limitation of elevation of the left eye in adduction. 
The patient was diagnosed as having Brown's syndrome. She was initially treated with indomethacin $50 \mathrm{mg}$ every eight hours for three weeks, but there was no benefit. She was then given prednisone, $20 \mathrm{mg}$ daily, with rapid resolution of the diplopia within $\mathbf{4 8}$ hours. Over the next few weeks the dose was gradually tapered and one year after starting prednisone she is free of symptoms, except for occasional diplopia on extreme upward gaze, and receives $7 \cdot 5 \mathrm{mg} /$ day prednisone.

\section{Discussion}

In 1950 Brown described the superior oblique tendon sheath syndrome, subsequently known as Brown's syndrome. ${ }^{1}$ It is the most common cause of an isolated apparent palsy of the inferior oblique muscle.

The oblique muscles have a complementary action in moving the eyes in the vertical plane. Normally, when the inferior oblique muscle moves the globe upward and inward, the superior oblique muscle relaxes. If the superior oblique tendon cannot lengthen or slide freely, the affected eye cannot be raised completely in full adduction. The patient reports diplopia on upward gaze.

Various theories have been proposed about the nature of the abnormality. Brown initially suggested that the syndrome was a developmental anomaly following congenital paralysis of the inferior oblique muscle, producing a contracture of its check ligament, the sheath of the anterior segment of the superior oblique. ${ }^{1}$ Later, as a attempt to explain specifically the intermittent cases, those associated with a clicking sound or sensation and those showing spontaneous resolution, it was suggested that there is a circumferential constriction of the trochlea and sheath preventing a locally enlarged tendon from sliding freely in the sheath. ${ }^{2-4}$ The various theories of the aetiology of the syndrome were well reviewed by Sandford-Smith, 5 who was the first to suggest the marked anatomical and clinical similarities between the tendon sheath syndrome and stenosing tenosynovitis. ${ }^{4}$

Mein reviewed 49 cases of Brown's syndrome and found that swelling of the posterior part of the superior oblique tendon was the most common mechanism, and also considered stenosing tenosynovitis to be the most likely cause of the tendon sheath syndrome. ${ }^{6}$

Despite the common association of tenosynovitis at other sites with rheumatoid arthritis (for example, trigger finger), this particular complication has only been reported in a few patients with rheumatoid arthritis, ${ }^{4}{ }^{7-11}$ juvenile chronic arthritis, ${ }^{10-14}$ or adult Still's disease. ${ }^{15}$ The disorder is usually self limited and transitory, and specific treatment is not usually required. Some cases have resolved with systemic steroid treatment, however, ${ }^{9-12} 15$ and an adult patient with rheumatoid arthritis with bilateral symptoms of Brown's syndrome showed a resolution of symptoms only after local corticosteroid injections into the region of the trochlea. ${ }^{8}$

Brown's syndrome has been also described in association with various non-rheumatic disorders including frontal sinusitis or frontal sinus surgery, ${ }^{16}$ trauma, ${ }^{17}$ focal metastatic lesions, ${ }^{18}$ Sneddon's syndrome, ${ }^{19}$ and hypogammaglobulinaemia. ${ }^{20}$

Although swelling of the superior oblique tendon has occasionally been noted on computed tomography scanning of the orbits, ${ }^{14} 1520$ in our patient computed tomography and magnetic resonance imaging findings were normal. The resolution of Brown's syndrome associated with the use of steroids, however, points to a tenosynovitis of the superior oblique tendons as the cause, though definitive proof is lacking. A true and selective palsy of the two inferior oblique muscles is exceptional.

Diplopia in patients with SLE has generally been attributed to nervous system disease. In our opinion, it is important to consider Brown's syndrome as a possible cause of diplopia in patients with SLE.

1 Brown $\mathrm{H}$ W. Congenital structural muscle anomalies. In: Allen J H, ed. Strabismus ophthalmic symposium I. St Louis: CV Mosby, 1950: 205-6.

2 Girard L J. Pseudoparalysis of the inferior oblique muscle. South Med F 1956; 49: 342-6.

3 Goldstein J H. Intermittent superior oblique tendon sheath syndrome. Am f Ophthalmol 1969; 67: 960-2.

4 Sandford-Smith J H. Intermittent superior oblique tendon sheath syndrome. A case report. Br $\mathcal{F}$ Ophthalmol 1969; 53: 412-7.

5 Sandford-Smith J H. Superior oblique tendon sheath syndrome and its relationship to stenosing tenosynovitis. $\mathrm{Br} \mathcal{J}$ Ophthalmol 1973; 57: 859-65.

6 Mein J. Superior oblique tendon sheath syndrome. Br Orthop 7 1971; 28: $70-6$.

7 Sims J. Acquired apparent superior oblique tendon sheath syndrome. Br Orthop $\mathcal{f}$ 1971; 28: 112-5.

8 Beck $M$, Hickling $P$. Treatment of bilateral superior oblique tendon sheath syndrome complicating rheumatoid arthritis. Br F Ophthalmol 1980; 64: 358-61.

9 Cooper C, Kirwan J R, McGill N W, Dieppe P A. Brown's syndrome: an unusual ocular complication of rheumatoid arthritis. Ann Rheum Dis 1990; 49: 188-9.

10 Calabozo M, Medina J, Diaz-Miguel C, Mohino-Cruz A. Síndrome de Brown asociado a artritis. Med Clin (Barc) 1988; 91: 220-2.

11 Killian P J, McClain B, Lawless O J. Brown's syndrome. An unusual manifestation of rheumatoid arthritis. Arthritis Rheum 1977; 20: 1080-4

12 Kemp A S, Searle C, Horne S. Transient Brown's syndrome in juvenile chronic arthritis. Ann Rheum Dis 1984; 43: 764 in $j$.

13 Wang F M, Wertenbaker C, Behrens M M, Jacobs J C. Acquired Brown's syndrome in children with juvenile rheumatoid arthritis. Ophthalmology 1984; 91: 23-6.

14 Roifman C M, Lavi S, Moore A T, Morin D J, Stein L D Gelfand $E$ W. Tenosynovitis of the superior oblique muscle (Brown's syndrome) associated with juvenile rheumatoid arthritis. F Pediatr 1985; 106: 617-9.

15 Kaufman L D, Sibory P A, Anand A K, Gruber B L. Superior oblique tenosynovitis (Brown's syndrome, as a manifestation of adult Still's disease. $\mathcal{F}$ Rheumatol 1987; 14: manifest.

16 Parks N M, Brown M. Superior oblique tendon sheath syndrome of Brown. Am $\mathcal{J}$ Ophthalmol 1975; 79: 82-6.
Brown H W. True and simulated superior oblique tendon Brown H W. True and simulated superior oblique tendo
sheath syndrome. Doc Ophthalmol 1973; 34: 123-36. sheath syndrome. Doc Ophthalmol 1973; 34: 123-36.
8 Slavin M L, Goodstein S. Acquired Brown's syndrome caused by focal metastasis to the superior oblique muscle. Am 7 Ophthalmol 1987; 103: 598-9.

19 Gato A, Ballesteros P, Alcaraz M, Villar M. Síndromes de Sneddon y Brown: una asociación no descrita. Med Clin (Barc) 1990; 95: 58.

20 Binkley K, Shore A, Buncic R, Roifman C. Acquired Brown's syndrome associated with hypogammaglobulinemia. I Rheumatol 1991; 18: 139-41. 